\title{
ON SOME COMPACT ALMOST KÄHLER LOCALLY SYMMETRIC SPACE
}

\author{
TAKASHI OGURO \\ Department of Mathematics and Information Science \\ Graduate School of Science and Technology \\ Niigata University \\ Niigata, 950-21, Japan
}

(Received April 8, 1996 and in revised form May 3, 1997)

\begin{abstract}
In the framework of studying the integrability of almost Kähler manifolds, we prove that if a compact almost Kähler locally symmetric space $M$ is a weakly *-Einstein manifold with non-negative *-scalar curvature, then $M$ is a Kähler manifold.
\end{abstract}

KEY WORDS AND PHRASES: Almost Kähler manifolds, locally symmetric spaces and weakly $*$-Einstein manifolds.

1991 AMS SUBJECT CLASSIFICATION CODES: 53C25, 53C35, 53C55.

\section{INTRODUCTION}

An almost Hermitian manifold $M=(M, J, g)$ is called an almost Kähler manifold if the corresponding Kähler form is closed, or equivalently $\underset{X, Y, X}{\mathfrak{S}} g\left(\left(\nabla_{X} J\right) Y, Z\right)=0$ for all $X, Y$, $Z \in \mathfrak{X}(M)$, where $\mathfrak{X}(M)$ denotes the Lie algebra of all smooth vector fields on $M$ and ${ }_{X, Y, Z}^{\mathfrak{S}}$ denotes the cyclic sum with respect to $X, Y, Z$. By the definition, a Kähler manifold $(\nabla J=0)$ is necessarily an almost Kähler manifold. It is well-known that if the almost complex structure of an almost Kähler manifold $M$ is integrable, then $M$ is a Kähler manifold. A non-Kähler, almost Kähler manifold is called a strictly almost Kähler manifold. Several examples of strictly almost Kähler manifolds have been constructed ([1], [6], [9], [13] and so on).

Concerning the integrability of almost Kähler manifolds, the following conjecture by Goldberg is known ([4]).

CONJECTURE. The almost complex structure of a compact almost Kähler Einstein manifold is integrable.

About the above conjecture, some progress have been made under some additional curvature conditions. For example, Sekigawa proved that the conjecture is true if the scalar curvature is non-negative ([11]). Further result have been obtained ([2], [3], [7], [8], [10], [12] and so on).

On any almost Hermitian manifold, we can define Ricci *-tensor, an analogue of the Ricci tensor, but involving the almost complex structure (see (2.1) below for the definition). On a Kähler manifold, the Ricci tensor and the Ricci *-tensor coincide. Therefore, it is natural to consider star-version of the Goldberg conjecture. An almost Hermitian manifold is called weakly *-Einstein if the Ricci *-tensor is a (not necessarily constant) multiple of the metric 
and *-Einstein if the Ricci *-tensor is a constant multiple of the metric.

In the present paper, concerning the star-version of the Goldberg conjecture, we shall prove the following.

THEOREM. Let $M=(M, J, g)$ be a compact almost Kähler locally symmetric space which is a weakly *-Einstein manifold with non-negative *-scalar curvature. Then, $M$ is a Kähler manifold.

The author express his sincere thanks to Prof. K. Sekigawa for his many valuable advice.

\section{PRELIMINARIES}

Let $M=(M, J, g)$ be a $2 n$-dimensional almost Hermitian manifold with the almost Hermitian structure $(J, g)$ and $\Omega$ the corresponding Kähler form of $M$ defied by $\Omega(X, Y)=g(X, J Y)$ for $X, Y \in \mathfrak{X}(M)$. We assume that $M$ is oriented by the volume form $d M=\frac{(-1)^{n}}{n !} \Omega^{n}$. Let $\nabla$ be the Riemannian connection and $R$ its curvature tensor given by $R(X, Y) Z=$ $\left[\nabla_{X}, \nabla_{Y}\right] Z-\nabla_{[X, Y]} Z$ for $X, Y, Z \in \mathfrak{X}(M)$. We denote by $\rho$ and $\tau$ the associated Ricci tensor and the scalar curvature, respectively. Moreover, let $\rho^{*}$, respectively $\tau^{*}$, denote the Ricci *-tensor, respectively the *-scalar curvature, defined by

$$
\begin{aligned}
\rho^{*}(x, y)=g\left(Q^{*} x, y\right) & =\operatorname{trace}(z \mapsto R(x, J z) J y), \\
\tau^{*} & =\operatorname{trace} Q
\end{aligned}
$$

for $x, y, z \in T_{p} M$, the tangent space of $M$ at $p \in M$. By using the first Bianchi identity, we have easily

$$
\rho^{*}(x, y)=\frac{1}{2} \operatorname{trace}(z \mapsto R(x, J y) J z) .
$$

Thus, in general, $\rho^{*}$ is neither symmetric nor skew-symmetric. But it satisfies the following identify.

$$
\rho^{*}(X, Y)=\rho^{*}(J Y, J X)
$$

for any $X, Y \in \mathfrak{X}(M)$.

Let $\left\{e_{1}, \ldots, e_{2 n}\right\}$ be an orthonormal basis of $T_{p} M$ at any point $p \in M$. In the present paper, we shall adopt the following notational convention:

$$
\begin{gathered}
R_{i j k l}=g\left(R\left(e_{i}, e_{j}\right) e_{k}, e_{l}\right), R_{i j k l}=g\left(R\left(J e_{\imath}, e_{\jmath}\right) e_{k} . e_{l}\right), \cdots, R_{i j k l}=g\left(R\left(J e_{\imath}, J e_{\jmath}\right) J e_{k}, J e_{l}\right), \\
\rho_{\imath \jmath}=\rho\left(e_{\imath}, e_{\jmath}\right), \ldots, \rho_{i j}=\rho\left(J e_{\imath}, J e_{j}\right), \quad \rho_{\imath \jmath}^{*}=\rho^{*}\left(e_{\imath}, e_{\jmath}\right), \ldots, \rho_{i j}^{*}=\rho^{*}\left(J e_{i}, J e_{j}\right), \\
J_{\imath \jmath}=g\left(J e_{\imath}, e_{\jmath}\right), \quad \nabla_{\imath} J_{j k}=g\left(\left(\nabla_{e_{1}} J\right) e_{\jmath}, e_{k}\right),
\end{gathered}
$$

and so on, where the latin indices run over the range $1,2, \ldots, 2 n$. Then, we have easily

$$
J_{i j}=-J_{j i}, \quad \nabla_{\imath} J_{j k}=-\nabla_{\imath} J_{k j}, \quad \nabla_{\imath} J_{j \bar{k}}=-\nabla_{\imath} J_{j k} .
$$

Now, we shall define smooth functions $A, B$ on $M$ respectively by

$$
\begin{aligned}
& A=\sum_{a, z, j, k, l=1}^{2 n}\left(\nabla_{a} J_{j i}\right)\left(\nabla_{a} J_{k l}\right) R_{i j k l}, \\
& B=\sum_{a, b, z, j, k, l=1}^{2 n}\left(\nabla_{a} J_{i j}\right)\left(\nabla_{a} J_{k l}\right)\left(\nabla_{b} J_{i j}\right) \nabla_{b} J_{k l},
\end{aligned}
$$


at any point $p \in M$.

Now, we assume that $M=(M, J, g)$ is an almost Kähler manifold. Then it is known that $M$ is a quasi-Kähler manifold, namely, the equality

$$
\nabla_{\imath} J_{j k}=-\nabla_{i} J_{j k}
$$

is valid. Thus, it follows immediately that $M$ is a semi-Kähler manifold, namely, the equality

$$
\sum_{a=1}^{2 n} \nabla_{a} J_{a i}=0
$$

is valid. We now recall the following curvature identity established by Gray ([5]):

$$
R_{\imath \jmath k l}-R_{\imath \jmath \bar{k} \bar{l}}-R_{i \bar{j} k l}+R_{\bar{i} \bar{j} \bar{k} \bar{l}}+R_{\bar{\imath} \bar{j} \bar{k} l}+R_{\bar{i} j k \bar{l}}+R_{\imath \bar{\jmath} k \bar{l}}+R_{i \bar{j} \bar{k} l}=2 \sum_{a=1}^{2 n}\left(\nabla_{a} J_{i j}\right) \nabla_{a} J_{k l} .
$$

From $(2.1) \sim(2.3)$ and $(2.6)$, we have easily

$$
\rho_{i j}^{*}+\rho_{j z}^{*}-\rho_{i j}-\rho_{i j}^{-\bar{j}}=\sum_{a, k=1}^{2 n}\left(\nabla_{a} J_{i k}\right) \nabla_{a} J_{j k}
$$

and further

$$
\|\nabla J\|^{2}=2\left(\tau^{*}-\tau\right)
$$

On one hand, transvecting $\sum_{b}\left(\nabla_{b} J_{\imath \jmath}\right) \nabla_{b} J_{k l}$ with (2.6) and taking account of (2.4), we have easily

$$
B=4 A \text {. }
$$

Further, we may easily observe that $M$ is Kähler if and only if $B=0$ holds identically on $M$.

\section{PROOF OF THEOREM}

Let $M=(M, J, g)$ be a $2 n$-dimensional compact almost Kähler locally symmetric space which is weakly *-Einstein with $\tau^{*} \geq 0$. We define a smooth vector field $\xi$ on $M$ by

$$
\xi=\sum_{a=1}^{2 n}\left(\sum_{i, j, k, l=1}^{2 n} R_{\imath \jmath k l} J_{i j} \nabla_{a} J_{k l}\right) e_{a}
$$

at each point $p \in M$. Then, taking account of $\nabla R=0$, the Ricci identity and (2.2), we have

$$
\begin{aligned}
\operatorname{div} \xi & =\sum_{a, z, j, k, l=1}^{2 n} \nabla_{a}\left(R_{\imath j k l} J_{\imath}, \nabla_{a} J_{k l}\right) \\
& =\sum R_{\imath \jmath k l}\left(\nabla_{a} J_{i j}\right) \nabla_{a} J_{k l}+\sum R_{i j k l} J_{\imath \jmath} \nabla_{a a}^{2} J_{k l} \\
& =A-\sum R_{\imath j k l} J_{\imath \jmath}\left(\nabla_{a k}^{2} J_{l a}+\nabla_{a l}^{2} J_{a k}\right) \\
& =A+\sum R_{\imath j k l} J_{\imath \jmath}\left(R_{a k l b} J_{b a}+R_{a k a b} J_{l b}+R_{a l a b} J_{b k}+R_{a l k b} J_{a b}\right) \\
& =A-\sum R_{\imath j k l} J_{\imath \jmath} R_{a b l k} J_{a b}-2 \sum R_{\imath \jmath k l} J_{\imath \jmath} J_{l b} \rho_{k b} \\
& =A+4 \sum\left(\rho_{\imath \jmath}^{*}\right)^{2}-4 \sum \rho_{i j}^{*} \rho_{\imath \jmath} .
\end{aligned}
$$


Since $M$ is weakly $*$-Einstein, from (3.1), we have

$$
\operatorname{div} \xi=A+\frac{2}{n} \tau^{*}\left(\tau^{*}-\tau\right)
$$

Thus, form (2.7) and (2.8), we have finally

$$
\operatorname{div} \xi=\frac{1}{4} B+\frac{1}{n} \tau^{*}\|\nabla J\|^{2}
$$

Therefore, by Green's Theorem, we obtain a following integral formula:

$$
\int_{M}\left(n B+4 \tau^{*}\|\nabla J\|^{2}\right) d M=0 .
$$

Since $B \geq 0$ and $\tau^{*} \geq 0$, it must follow that $B=0$ holds identically on $M$, and hence $M$ is a Kähler manifold. This completes the proof of Theorem.

\section{REFERENCES}

[1] CORDERO, L. A., FERNANDEZ, M. and GRAY, A., "Examples of compact non-Kähler almost Kähler manifolds," Proc. Amer. Math. Soc. 95 (1985), 280-286.

[2] DRAGHICI, T., "On the almost Kähler manifolds with Hermitian Ricci tensor," Houston J. Math. 20 (1994), 293-298.

[3] _ , "On some 4-dimensional almost Kähler manifolds," Kodai Math. J. 18 (1995), $156-168$.

[4] GOLDBERG, S. I., "Integrability of almost Kähler manifolds," Proc. Amer. Math. Soc. 21 (1969), 96-100.

[5] GRAY, A., "Curvature identities for Hermitian and almost Hermitian manifolds," Tôhoku Math. J. 28 (1976) 601-612.

[6] JELONEK, W., "Some simple examples of almost Kähler non-Kähler structures," to appear in Math. Ann.

[7] MURAKOSHI, N., OGURO, T. and SEKIGAWA, K., "Four-dimensional almost Kähler locally symmetric spaces," to appear in Differential Geometry and its applications.

[8] OGURO, T. and SEKIGAWA, K., "Non-existence of almost Kähler structure on hyperbolic spaces of dimension 2n( $\geq 4)$," Math. Ann. 350 (1994), 317-329.

[9] _ , "Almost Kähler structures on the Riemannian product of a 3-dimensional hyperbolic space and a real line," to appear in Tsukuba J. Math.

[10] SEKIGAWA, K., "On some 4-dimensional compact almost Hermitian manifolds," J. Ramanujan Math. Soc. 2 (1987), 101-116.

[11] _ _ "On some compact Einstein almost Kähler manifolds," J. Math. Soc. Japan 39 (1987), 677-684.

[12] SEKIGAWA, $\mathrm{K}$ and VANHECKE, L., "Four-dimensional almost Kähler Einstein manifolds," Ann. Math. Pura Appl. 157 (1990), 149-160.

[13] THURSTON, W. P., "Some simple examples of symplectic manifolds," Proc. Amer. Math. Soc. 55 (1976), 467-468. 


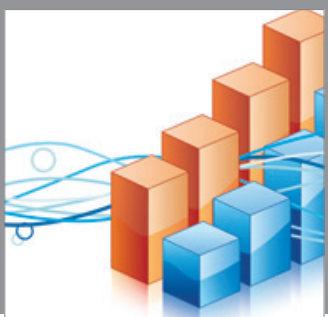

Advances in

Operations Research

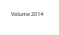

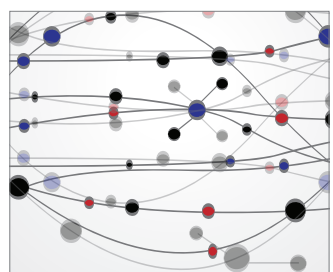

\section{The Scientific} World Journal
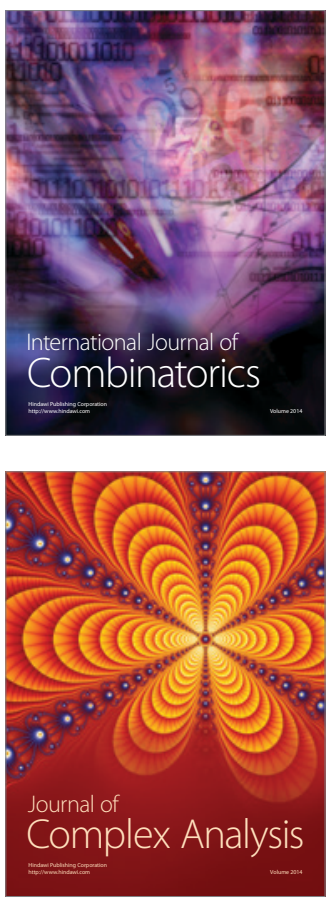

International Journal of

Mathematics and

Mathematical

Sciences
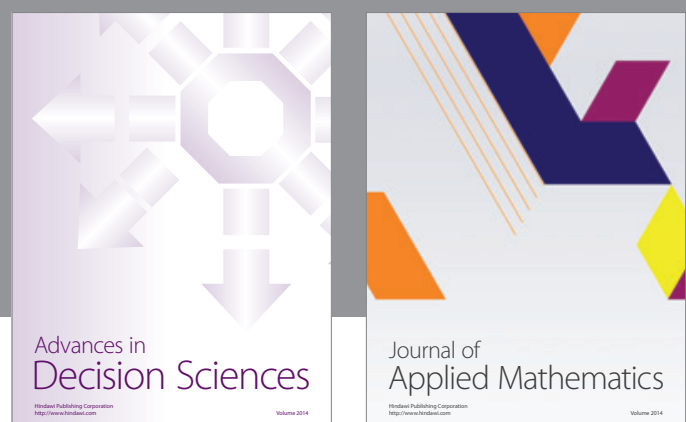

Journal of

Applied Mathematics
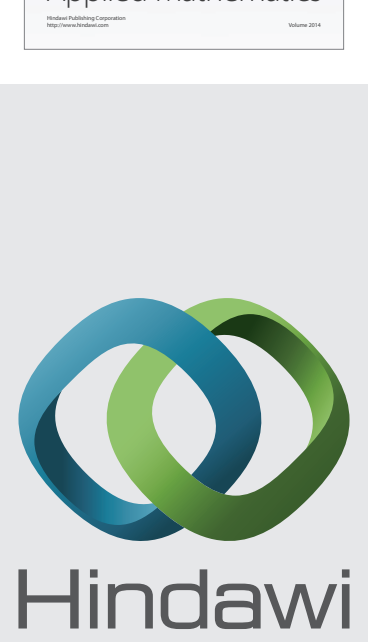

Submit your manuscripts at http://www.hindawi.com
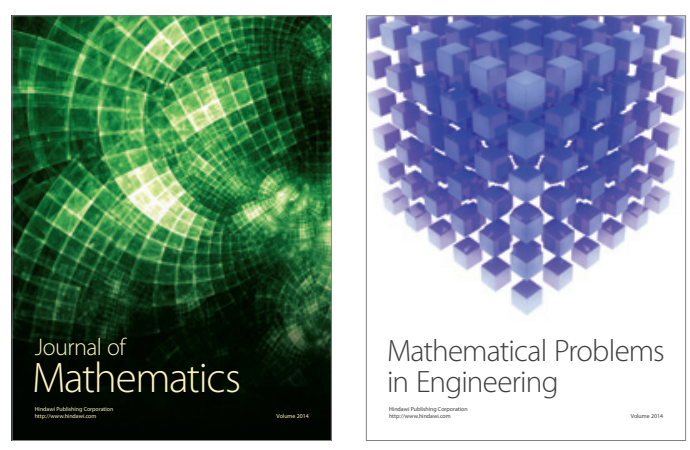

Mathematical Problems in Engineering
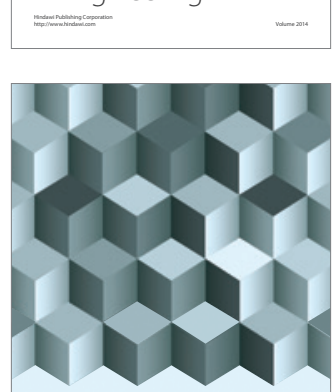

Journal of

Function Spaces
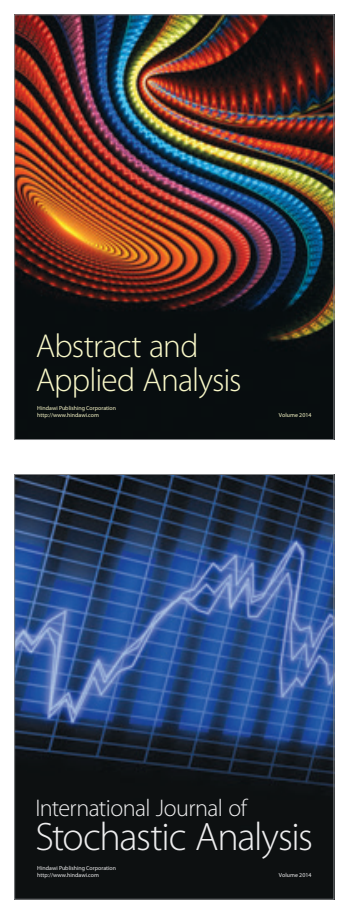

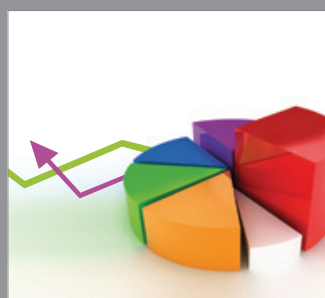

ournal of

Probability and Statistics

Promensencen
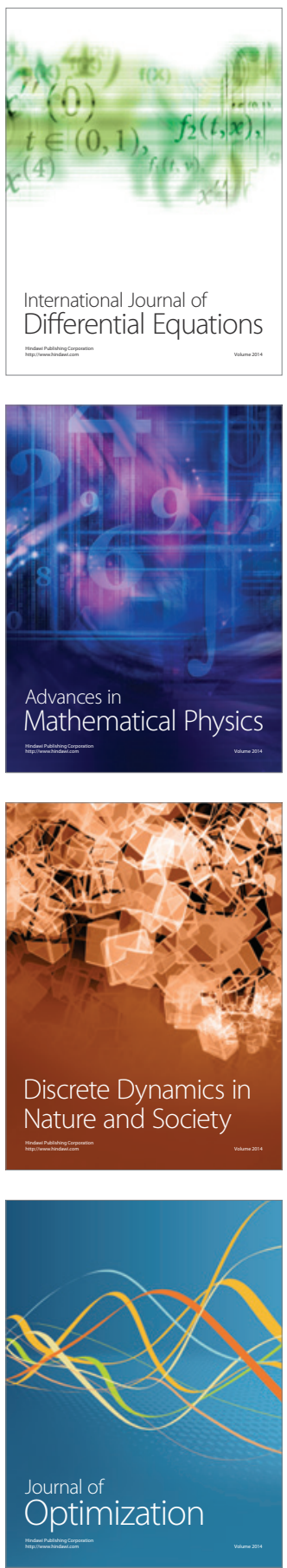